\title{
Fibre optic chemical sensor based on graphene oxide-coated long period grating
}

\author{
Chen Liu', Qi Cai ${ }^{\mathrm{a}, \mathrm{b}}$, Zhongyuan Sunc, Baojian Xub ${ }^{\mathrm{b}}$, Jianlong Zhao ${ }^{\mathrm{b}}$, Lin Zhang ${ }^{\mathrm{c}}$, Xianfeng Chen* ${ }^{\mathrm{a}}$ \\ ${ }^{a}$ School of Electronic Engineering, Bangor University, Bangor LL57 1UT, United Kingdom \\ ${ }^{\mathrm{b}}$ Shanghai Institute of Microsystem and Information Technology, Chinese Academy of Sciences \\ ${ }^{c}$ Aston Institute of Photonic Technologies, Aston University, Birmingham, United Kingdom
}

\begin{abstract}
In this work, a graphene oxide-coated long period fibre grating (GO-LPG) is proposed for chemical sensing application. Graphene oxide (GO) has been deposited on the surface of long period grating to form a sensing layer which significantly enhances the interaction between LPG propagating light and the surrounding-medium. The sensing mechanism of GO-LPG relies on the change of grating resonance intensity against surrounding-medium refractive index (SRI). The proposed GO-LPG has been used to measure the concentrations of sugar aqueous solutions. The refractive index sensitivities with $99.5 \mathrm{~dB} /$ RIU in low refractive index region (1.33-1.35) and $320.6 \mathrm{~dB} / \mathrm{RIU}$ in high index region (1.42-1.44) have been achieved, showing an enhancement by a factor of 3.2 and 6.8 for low and high index regions, respectively. The proposed GO-LPG can be further extended to the development of optical biochemical sensor with advantages of high sensitivity, real-time and label-free sensing.
\end{abstract}

Keywords: Long period grating; Graphene oxide; APTES salinization; Chemical sensor.

\section{INTRODUCTION}

Optical chemical sensors play a significant role in medicine, life science, security, food and environmental monitoring. Over the past decades, fibre optic sensing technology has been developed for qualitative and quantitative analysis in biochemistry due to the unique properties of small size, light weight and immunity to electromagnetic interference, and the additional advantages of high sensitivity, multiplex, label-free, real-time and in vivo detection ${ }^{[1,2]}$. Various fibre optic sensors have been proposed for biochemical sensing by using long period gratings (LPGs), tilted fibre gratings (FTGs), micro fibre Bragg gratings (mFBGs) ${ }^{[3,4]}$. The mechanism of fibre optic chemical sensing is based on evanescent field interactions between propagating light of fibre devices and the surrounding-medium ${ }^{[5,6]}$. In order to improve the surrounding-medium refractive index (SRI) sensitivity, several approaches have been proposed, such as using speciality D-shaped fibre, etching the cladding, polishing and tapering the fibre ${ }^{[7,8]}$. An alternative approach for the enhancement of of RI sensitivity has also been reported by depositing a nanoparticles thin film over the fibre gratings with RI higher than that of fibre cladding ${ }^{[9-11]}$.

Graphene oxide (GO) is a 2D nanomaterials and rich of oxygen-containing functional groups, which allows interaction between $\mathrm{GO}$ and a vast range of organic and inorganic materials. Hence, GO has attracted great interest for its exceptional electronic and photonic properties ${ }^{[12-14]}$. Due to its superior scalability and flexible processability of singlelayer sheets in aqueous or organic media, GO can be deposited on optical fibre surface in the form of thin film by dipcoating or chemical bonding techniques. To date, GO has been widely used in the fields of optoelectronics. The rich functional groups on GO surface readily make it possible to immobilize biomolecules and hold great potential for biochemical applications ${ }^{[15]}$.

In this work, we report a novel chemical sensor based on GO-coated LPG. The GO has been chemically immobilised on the fibre sensor surface via (3-aminopropyl) triethoxysilane (APTES) to provide better reliability and homogeneity. The GO-coated LPG has been used to measure the concentrations of sugar aqueous solutions with strong resonance intensity response, demonstrating high sensitivity of the variations of surrounding-medium refractive index.

*x.chen@bangor.ac.uk; phone 441248 382480; fax 441248361429

Sixth European Workshop on Optical Fibre Sensors, edited by Elfed Lewis, Proc. of SPIE Vol. 9916, 99160V · (C) 2016 SPIE · CCC code: 0277-786X/16/\$18 · doi: 10.1117/12.2235671

Proc. of SPIE Vol. $991699160 \mathrm{~V}-1$ 


\section{SENSING PRINCIPLE OF GO-COATED LPG}

A long period grating is formed typically by photoinducing a periodic refractive index modulation in the order of hundreds of micrometres in the fibre core, which promotes the light coupling from the fundamental core mode to a set of forward-propagating cladding modes. The working principle of grating attenuation intensity response to SRI changes using GO-coated LPG is based on the transmission equation ${ }^{[3]}: T_{\mathrm{i}}=1-\sin ^{2}\left(\kappa_{i} L\right)$, where $T_{\mathrm{i}}$ represents the minimum transmission of the $i$ th cladding mode, $\kappa_{i}$ is the coupling coefficient of the $i$ th cladding mode which is proportional to the overlap of electromagnetic field of fundamental core mode and the $i$ th cladding mode. $L$ is the length of the grating. External perturbation affects the evanescent field surrounding the LPG hence changes the coupling coefficient, yielding the measurable intensity change of LPG attenuation peaks.

\section{DEPOSITION OF GRAPHENE OXIDE AND CHARACTERISATION}

The $15 \mathrm{~mm}$-long LPGs with period of $400 \mu \mathrm{m}$ were $\mathrm{UV}$-inscribed in $\mathrm{H}_{2}$-loaded single mode fibre by the use of a pointby-point technique. After the UV exposure, the gratings were annealed at $85^{\circ} \mathrm{C}$ for $24 \mathrm{~h}$ to remove residual hydrogen and stabilize their optical properties. Prior to GO deposition, the LPG was cleaned with methanol to reduce the residual contaminant on fibre surface, followed by immersion in $1.0 \mathrm{M} / \mathrm{L} \mathrm{NaOH}$ solution for $60 \mathrm{~min}$ at room temperature to make the fibre surface hydrophilic and improve the intensity of -OH group on the fibre surface, washed with deionized (DI) water thoroughly and dried. Splenisation of the LPG surface was performed by immersing the cleaned LPG in fresh $5 \%$ APTES solution ( $\mathrm{v} / \mathrm{v}$ in ethanol) for $12 \mathrm{~h}$ at room temperature. The APTES-modified LPG was baked at $70^{\circ} \mathrm{C}$ for $30 \mathrm{~min}$ then was inserted in $1.0 \mathrm{mg} / \mathrm{L} \mathrm{GO}$ aqueous solution for $2 \mathrm{~h}$ at room temperature, and baked at $70{ }^{\circ} \mathrm{C}$ for $30 \mathrm{~min}$. The processes of APTES salinization (for $2 \mathrm{~h}$ ) associated with GO deposition $(2 \mathrm{~h}$ ) were further repeated three cycles to realize homogeneous $\mathrm{GO}$ deposition on fibre surface.

Characteristics of graphene oxide deposited on LPG surface were verified. Fig. 1a shows the image of GO layer coated on the fibre surface. Fig. 1b plots the Raman spectra of bare fibre and GO-coated fibre by using Renishaw Raman Microscope 1000 (with $633 \mathrm{~nm}$ light). The significant peaks (D and G) were observed for GO-coated LPG. The D peak at $1335 \mathrm{~cm}^{-1}$ was assigned to local defect and disorder of graphene oxide caused by attachment of hydroxyl and epoxide groups on the carbon basal plane and edges. The $G$ peak around $1599 \mathrm{~cm}^{-1}$ was due to the first order scattering of the $E_{2 \mathrm{~g}}$ phone of $\mathrm{sp}^{2}$ carbon atoms ${ }^{[15]}$. In further, the LPG spectra were monitored before and after the GO deposition (Fig. 1c), which induced a blue shift of $3.8 \mathrm{~nm}$ and $12 \mathrm{~dB}$ intensity change of LPG attenuation peak. All these results indicated that the graphene oxide was successfully coated onto fibre surface.

(a)

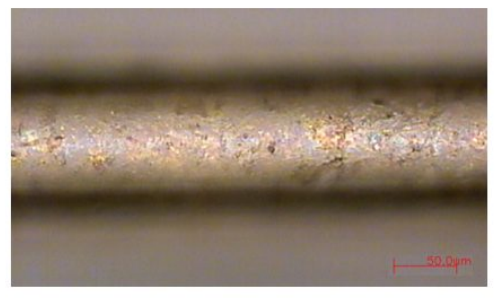

(c)

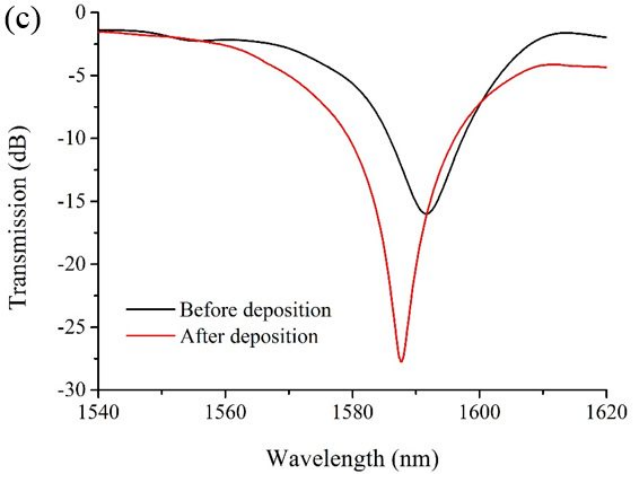

(b)

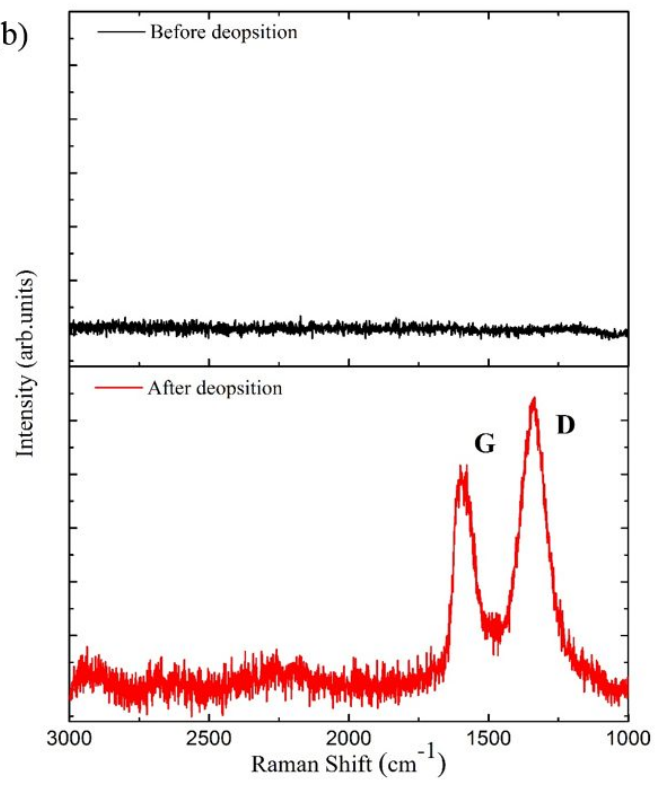

Fig.1 (a) Microscope image of GO-coated LPG. (b) Raman spectra of fibre samples. (c) LPG spectra before and after GO deposition. 


\section{SUGAR CONCENTRATION MEASUREMENT}

The experiment setup is shown in Fig. 2. In the real time monitoring system, broadband light sources (EDFA CPE1437, Nortel Networks Inc.) were used along with an optical spectrum analyser (OSA, Agilent HP86140, Agilent Technologies Inc., range $600 \mathrm{~nm}$ to $1700 \mathrm{~nm}$ ). The OSA was connected to a computer and the optical spectra were captured by a customized program.

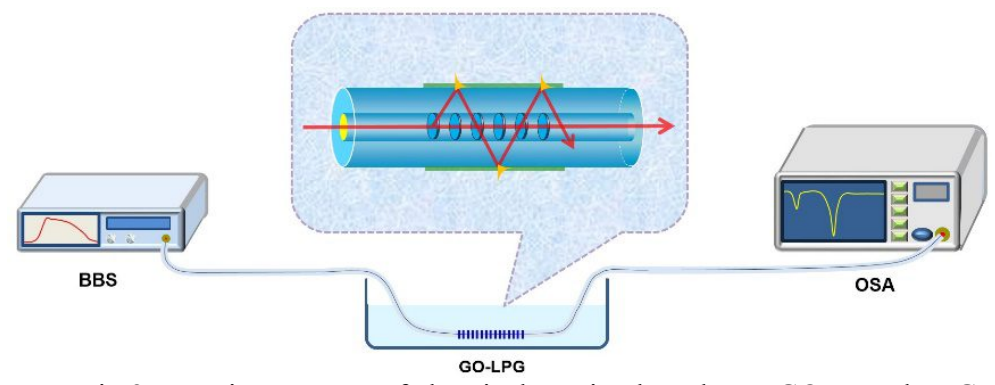

Fig.2 Experiment setup of chemical sensing based on a GO coated LPG.

The GO-coated LPG was used for the implementation of an optical chemical sensor to measure the sugar aqueous solutions with concentrations ranging from $0 \%$ to $60 \%$. Measured by Abbe refractometer, the refractive indices of sugar solutions increase more-or-less linearly from 1.331 to 1.441 for sugar concentrations changing from $0 \%$ to $60 \%$. To minimize the thermal and bend cross sensitivity, the grating has been placed in a straight V-groove holder, immersed in the sugar solution, and under a constant room temperature environmental condition. A careful clean-and-dry process that incorporates washing of LPG in methanol was followed after each measurement to remove the residual sugar form both grating and holder substrate. It was ensured that the spectrum in air was recovered after each measurement before the subsequent measurement was continued.

(a)

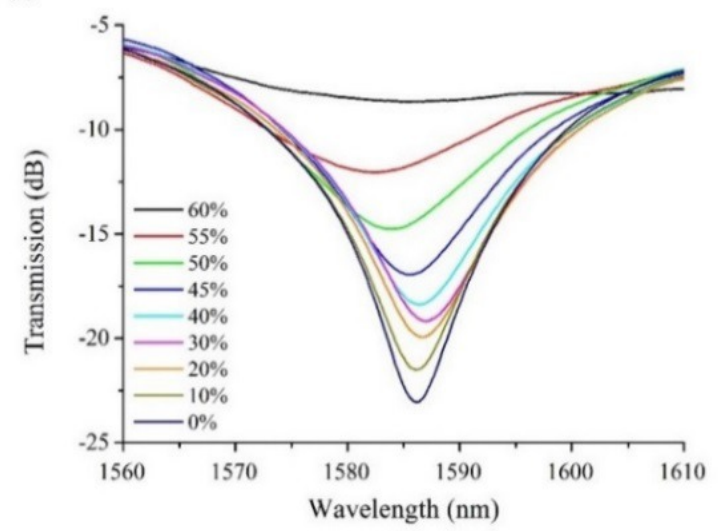

(b)

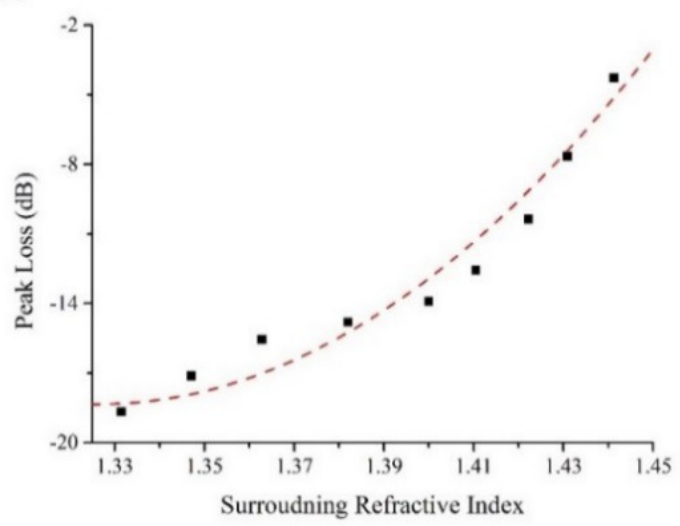

Fig.3 (a) Transmission spectra at different sugar concentration. (b) The attenuation loss against RI change.

Fig. 3a depicts the transmission spectra of the GO-LPG resonance at $1587.8 \mathrm{~nm}$. It can be seen clearly that the intensity of attenuation peak decrease with the increase of sugar concentrations whereas the slight variation in wavelength of the attenuation appear. These results are consistent with the reported carbon-nanotube-deposited LPG sensor ${ }^{[9]}$. As the real part refractive index of GO is higher than cladding, the cladding modes are performed as radiation modes in the presence of deposited GO layer. When chemical molecules, especially polar molecules, interact with the graphene oxide material altering the permittivity of graphene oxide and hence change the optical properties of $\mathrm{GO}^{[13]}$, inducing the coefficient of light coupling. Fig. 3b plots the intensity change of the attenuation peak against RI (1.331-1.441) of the sugar solutions. It is clear that the intensities of attenuation peak show a good monotonicity and a nonlinear relationship to the RI. The RI sensitivity achieves $99.5 \mathrm{~dB} / \mathrm{RIU}$ for low RI region (1.33-1.35) and 320.6 dB/RIU for high RI region (1.42-1.44), exhibiting more than 3.2 times and 6.8 times higher than previously reported carbon nanotube-deposited LPG for low and high RI regions, respectively ${ }^{[9]}$. In particular, the GO-coated LPG provides good RI sensitivity for low RI range (1.33-1.35) where bioassays and biochemical events are usually carried out. We calculate the chemical sensing performance of GO-coated LPG by converting the SRI sensitivity to the corresponding sugar concentration sensitivity. If the concentration sensitivity is defined as peak intensity change induced by $1 \%$ sugar concentration change, the maximum sensitivities are $0.16 \mathrm{~dB} / \%$ and $0.61 \mathrm{~dB} / \%$ for low and high concentration, respectively. 


\section{CONCLUSION}

We have presented a graphene oxide-coated LPG for sugar concentration measurement. The graphene oxide has been deposited on LPG surface via APTES as sensitisation material to enhance the interaction between fibre cladding modes to external medium. The characterisation of GO layer has been carried out by Raman microscope, optical microscope and the monitored LPG spectrum. The GO-LPG based sensor has been used to measure the sugar solution concentrations, demonstrating the enhanced RI sensitivities of $76.5 \mathrm{~dB} / \mathrm{RIU}$ in low RI region (1.33-1.35) and 320.6 $\mathrm{dB} / \mathrm{RIU}$ in high RI region (1.42-1.44). The high RI sensitivity in low region indicates that GO-coated LPG could be a good candidate for biochemical sensing application.

\section{ACKNOWLEDGEMENT}

The authors acknowledge the support from EU FP7 PIRSES-2013-612267 (iPhoto-Bio) and Sêr Cymru NRN097.

\section{REFERENCES}

1. Wang, X. and Wolfbeis, O. S., "Fiber-Optic Chemical Sensors and Biosensors (2013-2015)," Anal. Chem., 88, 203-227 (2016).

2. Fan, X. and White, I. M. "Optofluidic microsystems for chemical and biological analysis," Nat. Photonics, 5, $591-597$ (2011).

3. Erdogan, T., "Cladding mode resonances in short and long period fiber grating filters,” J. Opt. Soc. Am., 14, 1760-1773 (1997).

4. Albert, J., Shao, and L.-Y., Caucheteur, C., "Tilted fiber Bragg grating sensors,” Laser Photonics Rev., 7, 83-108 (2013).

5. James, S. W. and Tatam, R. P., “Optical fibre long-period grating sensors: characteristics and application,” Meas. Sci. Technol., 14, 49-61 (2003).

6. Shu, X., Zhang, L., and Bennion, I., "Sensitivity characteristics of long-period fiber," J. Lightwave Technol., 20, 255-266 (2002).

7. Jang, H. S., Park, K. N., Kim, J. P., Sim, S. J., Kwon, O. J., Han, Y. G., and Lee, K. S. "Sensitive DNA biosensor based on a longperiod grating formed on the side-polished fiber surface," Opt. Express., 17, 3855-3860 (2009).

8. Chen. X., Zhou, K., Zhang, L. and Bennion, I.., "Simultaneous measurement of temperature and external refractive index by use of a hybrid grating in D fiber with enhanced sensitivity by HF etching,” Appl. Opt., 44, 178-182 (2005)

9. Tan, Y. C., Ji, W. B., Mamidala V., Chow K. K., and Tjin S. C., "Carbon-nanotube-deposited long period fiber grating for continuous refractive index sensor applications,” Sens. Actuators B. Chem., 196, 260-264 (2014)

10. Cusano, A., Iadicicco, A., Pilla, P., Contessa, L., Campopiano, S., Cutolo, A., and Giordano, M., "Mode transition in high refractive index coated long period gratings," Opt. Express, 14, 19-34 (2006)

11. Sansone, L., Malachovska, V., La Manna, P., Musto, P., Borriello, A., De Luca G. and Giordano M., "Nanochemical fabrication of a graphene oxide-based nanohybrid for label-free optical sensing with fiber optics," S Sens. Actuators B. Chem., 202, 523-526 (2014).

12. Loh, K. P., Bao, Q., Eda, G., and Chhowalla, M., "Graphene oxide as a chemically tunable platform for optical applications," Nat. Chem., 2, 1015-1024 (2010).

13. Schedin, F., Geim, A. K., Morozov, S. V., Hill, E. W. , Blake, P., Katsnelson M. I. and Novoselov K. S., "Detection of individual gas molecules adsorbed on graphene," Nat. Mater., 6, 652-655 (2007).

14. Zhu, Y., Murali, S., Cai, W., Li, X., Suk, J. W., Potts, J. R. and Ruoff, R. S., "Graphene and graphene oxide: synthesis, properties, and applications," Adv. Mater., 22, 3906-3924 (2010).

15. Zhang, S. L., Du, Z., and Li, G. K., "Layer-by-layer fabrication of chemical-bonded graphene coating for solid-phase microextraction,” Anal. Chem., 83, 7531-7541 (2011). 